\title{
Evaluation of Keratoconus Progression after Intrastromal Corneal Ring Segments Implantation
}

\author{
Livia Cristina Rios ${ }^{1 *}$, Aristofanes Canamary $\mathrm{Jr}^{1,3}$, Henrique Figueiredo ${ }^{2}$, \\ Mariana Amaranto de Souza ${ }^{2}$, Pablo Felipe Rodrigues ${ }^{3}$, Rodrigo Teixeira \\ Santos $^{1,3}$ and Bernardo Kaplan Moscovici ${ }^{1,3}$ \\ ${ }^{1}$ Unidade Paulista de Oftalmologia, São Paulo, SP, Brazil \\ ${ }^{2}$ Fundação Hilton Rocha, Belo Horizonte, MG, Brazil \\ ${ }^{3}$ Unifesp, São Paulo, SP, Brazil \\ *Corresponding Author: Livia Cristina Rios, Unidade Paulista de Oftalmologia, São \\ Paulo, SP, Brazil.
}

\author{
Received: April 07, 2021 \\ Published: April 30, 2021 \\ (C) All rights are reserved by Livia Cristina \\ Rios., et al.
}

\begin{abstract}
Objectives: To report the evaluation of keratoconus progression after Ferrara intrastromal corneal ring segment implantation.

Materials and Methods: In this retrospective study, we evaluated progression in eyes with keratoconus after intrastromal corneal ring segments implant. It was considered progression when the increase of the maximum keratometry was greater than 1 diopter (D) when comparing the first postoperative exam ( 6 months), and the last exam.

Results: There was no progression after the surgery. when comparing the mean maximum keratometry pre-op (46.75D) and the pos op data (46.01D), with statistically significant (p-value: 0.047). An increase of less than $1 \mathrm{D}$ in these patients was also observed. In a descriptive analysis, 41 eyes were studied: 8 eyes had an increase greater than $1 \mathrm{D}, 19$ eyes had an increase equal or less than 1 D and 14 eyes maintained the initial D or had decreased within the years. Analyzing patients $\leq 21$ years, the variation of maximum keratometry between 6 months postoperatively was higher in this group (1.82 D \pm 3.11 ) than in the group with more than 21 years (0.5 D \pm 1.27$)$. This data was not statistically significant $(\mathrm{p}=0.148)$.
\end{abstract}

Conclusion: This study showed an evolution of maximum keratometry in the postoperative period of the intrastromal ring surgery. However, this increase in the stipulated time of the study was less than $1 \mathrm{D}$ in the majority of patients.

Keywords: Keratoconus; Corneal Diseases; Corneal Stroma; Cornea; Corneal Topography

\section{Introduction}

Keratoconus is a corneal ectatic disease characterized by noninflammatory progressive thinning of unknown cause in which the cornea assumes a conical shape [1]. This ectasia has a more important progression characteristic in teenagers and young adults. It can also cause irregular astigmatism, progressive myopia, protrusion resulting in decreasing the quality of vision [2]. Rigid gaspermeable contact lenses are used to provide a regular anterior surface that makes compensation of myopia and irregular astigmatism. Contact lens intolerance can make it difficult to fit lenses in eyes with a more advanced stage of disease especially in eyes with allergy and high sensibility. Therefore, surgery should be a viable option [3].

Intrastromal corneal ring segments (ICRS) have been used to reduce the corneal curvature and high order aberrations, and consequently, reduce and regularize corneal astigmatism and improving visual acuity. Besides, ICRS implantation is a surgical alternative to delay, if not eliminate, the need for lamellar or penetrating keratoplasty [2]. 
Many studies have evaluated the importance of the ICRS in improving visual acuity, others have compared the improvement in values such as maximum keratometry (Kmax), for example, by topography and tomography examinations.

We know that the method used to stabilize keratoconus progression is crosslinking (CXL). Corneal collagen cross-linking (CXL) with riboflavin and ultraviolet A (UVA) light is a method to surgically increase the rate of crosslinks within and between collagen fibers using photopolymerization [4]. The indication for this procedure is the progression of the disease evidenced by corneal topography [5].

\section{Objective of the Study}

The objective of this study is to investigate the interference of the intrastromal ring in the progression of keratoconus in patients who have never undergone other surgical procedures, including CXL.

\section{Methods}

A retrospective chart analysis study was performed with patients diagnosed with keratoconus at a private ophthalmology clinic in Belo Horizonte, Minas Gerais.

The study was approved by the Ethics Committee of the Instituto Suel Abujamra (Plataforma Brasil), CAAE 36902220.8.0000.5477 and followed the tenets of the Declaration of Helsinki.

\section{Patient selection}

The inclusion criteria were age between 15 to 30 years; keratoconus, regardless of stages, according to Rabinowitz criteria: central keratometry above 47.2 D, corneal topographic inferior-superior asymmetry $>1.4 \mathrm{D}$ or irregular topographic astigmatism [1-3].

The exclusion criteria were previous ocular surgeries or trauma; ocular surface disorders; corneal opacities; diabetes; use of oral medication or hormones that could interfere in corneal surface or curvature; systemic diseases; pregnancy, history of contact lenses wearing 72 hours before the topographic examination. Only subjects who had a collection made in a period of 6 months were selected, so we analyzed 33 charts, excluded 8 patients and our sample size was 25 patients.

\section{Surgical procedure}

All surgeries were performed by an experienced surgeon. The procedure was performed under sterile conditions. An eyelid speculum was used to expose the eye and the first Purkinke reflex was marked with a cannula while asking the patient to fixate on the corneal reflex of the microscope. A $5.0 \mathrm{~mm}$ tunnel and incision site was marked with gentian-violet ink. We used a diamond blade to perform the incision, at $80 \%$ of corneal thickness at the incision site, followed by the confection of a concentric stromal tunnel channel and consequent implantation of the segment (s).

\section{Pre and postoperative evaluation}

All patients underwent a complete eye examination, including evaluation of the anterior segment, intraocular pressure and fundoscopy.

The following data were analyzed: corrected distance visual acuity (CDVA) (logMAR scale), refractive cylinder, and keratometry data (k1 and $\mathrm{k} 2$ ). Both preoperative and last appointment data were compared. It was also compared the third-month postoperative keratometry to the final keratometry. Besides, the mean age of patients who presented progression of keratometry between the 6-month postoperative period and the last postoperative period was analyzed. The number of appointments was reduced over time as some patients did not attend some postoperative visits. All topographic data were obtained from a Scheimpflug Tomographer Pentacam (Oculus, Arlington, USA). All surgeries were performed by the same surgeon (RPS) and the Ferrara ICRS nomogram was used for ICRS selection (Ferrara Ring, Belo Horizonte, Brazil) in all cases.

\section{Post-operative care}

At the end of surgery therapeutic contact lens was placed and removed after 3 days in all patients.

The postoperative regimen consisted of moxifloxacin $0.5 \%$ (Vigamox; Alcon, Forth Worth, TX) and dexamethasone 0.1\% (Maxidex; Alcon, Forth Worth, TX) eye drops 4 times daily for 2 weeks.

\section{Data analysis}

We compared the average of Kmax of the axial map collected in the period of 6 months with the value of the last collection (inde- 
pendent of the period of the last collection). In this way, since we have paired data, we used the paired Student-T test. We recall that we are using parametric statistical tests since we tested the normality of the quantitative variables of the main outcome through the Kolmogorov-Smirnov (KS) test and concluded that there is a distribution of normality. Parametric tests are more powerful in detecting significance [8].

\section{Results}

We studied 25 patients, 13 men and 12 women with a mean age of $22 \pm 6$ years (ranging from 16 to 27 years). The follow up ranged from 15 to 90 months, mean of $39.1 \pm 15.63$ months. All descriptive data are available in table 1.

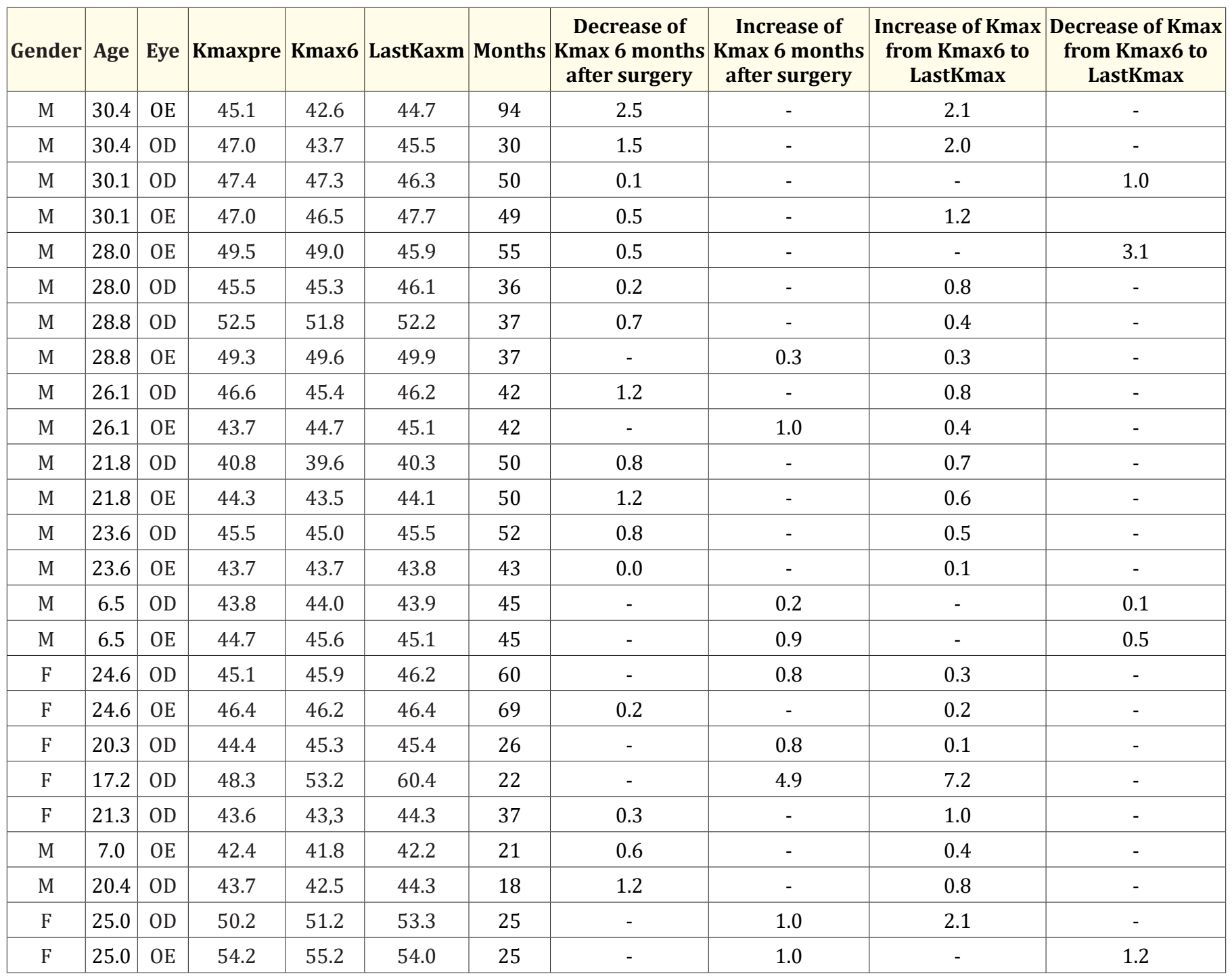

Table 1: Descriptive data of the study group.

Months: Months of follow-up after surgery; Kmax: Maximum Keratometry; Keratometry measured in diopters; Age measured in years; Kmaxpre: Maximum Keratometry before surgery; Kmax6: Maximum Keratometry 6 months after surgery; LastKmax: Maximum Keratometry in the last visit after surgery. 
We found a statistically significant mean difference of the Kmax, that is, the mean in the collection 6 months postoperatively was 46.01 versus 46.75 in the last collection ( $p$-value $=0.047$ ), although the mean increase was less than $1 \mathrm{D}$ diopter (Table 2).

We categorize the delta of the Kmax for these 25 subjects and we analyzed the distribution of the relative frequency (percentage) of increase (Table 3). We used the Equality of Two Proportions test [6].

\begin{tabular}{|c|c|c|}
\hline K max & 6 months & Last \\
\hline Average & 46,01 & 46,75 \\
\hline Median & 45,30 & 45,50 \\
\hline Standard deviation & 3,75 & 4,27 \\
\hline CV & $8 \%$ & $9 \%$ \\
\hline Min & 39,60 & 40,30 \\
\hline Max & 55,20 & 60,40 \\
\hline N & 25 & 25 \\
\hline IC & 1,47 & 1,67 \\
\hline P-value & 0,047 & \\
\hline
\end{tabular}

Table 2: Comparison of the maximum keratometry (Kmax) moments.

Subtitle: CV: Coefficient of Variation; Min: Minimum; Max: Maximum; N: Number; IC: Confidence Interval.

\begin{tabular}{|c|c|c|c|}
\hline Increase Kmax & $\mathbf{N}$ & $\mathbf{\%}$ & P-value \\
\hline No & 6 & $24,0 \%$ & $<0,001$ \\
\hline Yes & 19 & $76,0 \%$ & \\
\hline
\end{tabular}

Table 3: Distribution of maximum keratometry (Kmax) increase (from Kmax6 to LastKmax).

Kmax6: Maximum keratometry 6 months after surgery; LastKmax: Maximum keratometry in the last visit after surgery.

Thus, that there is statistical significance in the distribution of the Kmax increase, where $76.0 \%$ had an increase compared to only $24.0 \%$ that did not increase ( $\mathrm{p}$-value $<0.001$ ) (Graphic 1 ).

We found that there is an increase in mean values over time (with little infliction in the period of 6 to 12 months), but this evolution of mean values was not considered statistically significant.

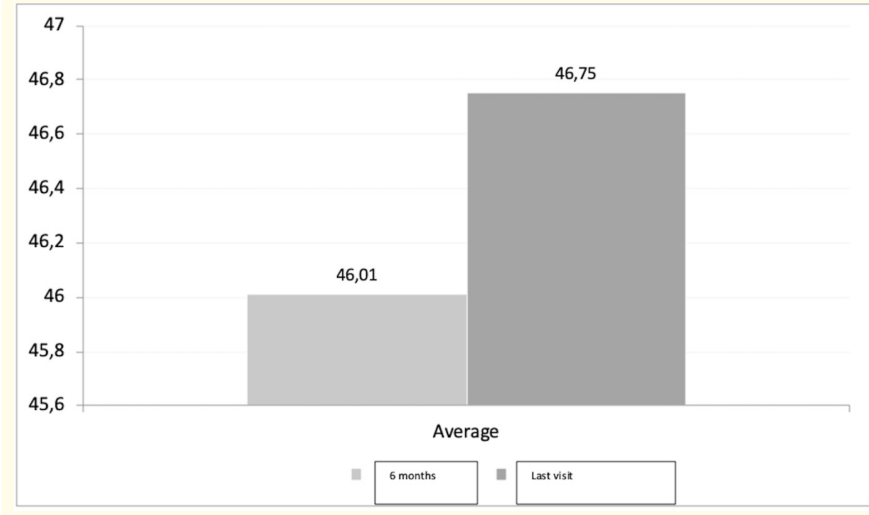

Graphic 1: Comparison of mean maximum keratometry in diopters.

Analyzing patients $\leq 21$ years, the variation of Kmax between 6 months postoperatively was higher in this group (1.82 D \pm 3.11 ) than in the group with more than 21 years $(0.5 \mathrm{D} \pm 1.27)$. This data was not statistically significant $(p=0.148)$, maybe due to the small sample. When we analyze the percentage of patients in these groups who had an increase greater than $1 \mathrm{D}$ over time, we found that $40 \%$ had an increase greater in the younger group and $36.8 \%$ in the older group, also not statistically significant.

A descriptive analysis is also important for the study. Of the 41 eyes studied, only 8 eyes had an increase equal to or greater than $1 \mathrm{D}, 19$ eyes had an increase smaller than $1 \mathrm{D}$ and 14 eyes maintained the Kmax or had a decrease of the value of Kmax. Of these 8 patients who increased Kmax after ICRS, the variation in Kmax increase was between 1 and $7.2 \mathrm{D}$, and the mean was $2.82 \mathrm{D}$, and the progression of 7.2 D was only found in one patient. We found a decrease of Kmax from 6 months to last visit in 5 patients, 2 with a decrease around 1 diopter and one with 3.1 diopters of decrease.

\section{Discussion and Conclusion}

Some reports suggest that ICRS implantation in keratoconic corneas can not only achieve visual rehabilitation but also perhaps stop the progression of the disease $[10,11]$. Our study did not evaluate visual rehabilitation after ICRS because there are several studies with these data in the literature, however 15 patients showed improvement in Kmax after 6 months of surgery, one showed no 
change and 9 showed worsening (possibly due to the progression of keratoconus). We chose to compare only Kmax, as it is one of the keratoconus progression indices and one of the simplest to collect and compare. Besides that, differential topographies, in retrospective studies, sometimes are not possible to use, due to errors in patient registration.

Studies tried to develop an artificial model of keratoconic eyes and they provided a hypothesis that in ectatic eyes there is a focal alteration in biomechanical in the thinnest and steeper area that generate a vicious circle of progression and with the implant of ICRS leads to flattening of the cornea and could halt this progression [3].

New biomechanic studies with Brillouin microscopy found that there is a focal weakening in the thinner and steeper are giving strength to these finite model thoughts [12].

Intrastromal ring segments can re-distribute corneal stress by shortening the lamellae and changing the shape of the cornea without altering intrinsic properties. This causes the cornea to derive viscoelastic progression and form a more regular cornea over time and the biomechanical injury caused by intraocular pressure, gravity or mechanical injury is more distributed and smoothed [13].

In contrast to this information, the ICRS implantation does not indicate stopping disease progression and CXL has been shown to stop the progression of the disease $[14,15]$.

De Araujo., et al. in a recent study, studied the progression of the disease after ICRS over 5 years. With 34 patients divided by age (more or less 21 years old) and the majority of the patients classified in stage 3 keratoconus, he found that patients under 21 years are more likely to show the progression of the disease $42 \%$ of them showed progression) than patients with more than 21 years (7\% of progression) as well as the mean progression of Kmax in this group was less than 2D [17]. Our results were similar to the older patients group, but we did not find statistically significant values, maybe due to our smaller sample.

Vega-Estrada., et al. in a similar study with 18 eyes with a mean age of 25.75 years \pm 3.59 (SD), in patients with documented progression of the disease, implanted ICRS with good postoperatively results, but, from 6 months postoperatively to 5 years, the mean $\mathrm{K}$ value regressed $3.36 \mathrm{D}$, indicating the progression of the disease. We evaluated patients without documented progression, and we found less progression of the disease, may be due to that [18].

Moscovici., et al. in a similar study with 170 eyes, comparing keratoconus progression (measured by k1 and k2) after ICRS found that, approximately $40 \%$ showed increase in keratometry greater than one diopter (mean age 26 to 27 years) and $60 \%$ less than one diopter (mean age 28 to 29 years). Only three patients had progression more than 3 diopters. Our data corroborate the study cited. Although our sample is smaller, we studied Kmax which is more accurate to assess disease progression and our sample was younger, thus with a higher probability of increased keratoconus.

We observed in their study a low degree of progression of the disease after surgery, even in younger patients. However, rigorous follow-up is necessary within a short interval of time, the best visual acuity, and complementary exams, such as corneal tomography to help in the identification of the increase and variation of Kmax. Combination therapy of 2 procedures may also show good results, for example, ICRS plus CXL can perform the redistribution of corneal stress via ICRS or else ablation with bio-mechanical change via CXL. Combinations have the potential to improve the shape of the cornea over time.

In this study, we compared patients with keratoconus before and after the surgical procedure of ring implantation to conclude whether or not they interfered in the evolution of the disease and how significant it is for ophthalmology science.

It is still uncertain the progression of keratoconus in the patients after ICRS. Our study has limitations as small sample size and it is not a double-blind randomized clinical trial and the value of the first measure of Kmax can still change up to 6 months postoperatively (on average). However, these data may show that in cases of patients with keratoconus, with low visual acuity and intolerance to the contact lens, the ICRS implant and subsequent analysis of disease progression, before performing CXL surgery, may show benefits, because not all patients presented the evolution of ectasia after ICRS implant surgery. 
Challenging cases with thin corneas in the keratonic area and thick in the 5-millimeter circle, that we fear to perform crosslinking even with documented progression, could be benefited by ICRS implant so as the rare cases that presents an increase of Kmax even after CXL that we could think of performing an ICRS implant before a second CXL surgery. It is important to note that there are still some patients who had an increase in Kmax after implantation of ICRS.

We found, after implantation of ICRS, that the majority of patients did not have increase of more than one diopter in Kmax. Since very few patients had a higher progression, ICRS implantation could be effective for slowing disease progression. Finally, double-blinded randomized studies analyzing more indexes should enlighten this field of study.

\section{Financial Disclosure}

No author has a financial or proprietary interest in any material or method mentioned.

\section{Bibliography}

1. Ferrara Paulo and Torquetti Leonardo. "Ferrara Ring". Chapter 16.

2. Jr Renato Ambrósio., et al. "Corneal Biomechanics in Ectatic Diseases: Refractive Surgery Implications". The Open Ophthalmology Journal 11.1-2 (2017): 176-193.

3. Roberts Cynthia J and Dupps Jr William J. "Biomechanics of corneal ectasia and biomechanical treatments". Journal of Cataract and Refractive Surgery - LWW Journals 40 (2014): 991998.

4. Chatzis Nico and Hafezi Farhad. "Progression of keratoconus and efficacy of corneal collagen cross-linking in children and adolescents". Journal of Refractive Surgery 38.11 (2012): 753758.

5. Pinero DP., et al. "Characterization of corneal structure in keratoconus". Journal of Cataract and Refractive Surgery - LWW Journals 38 (2012): 2167-2183.

6. Jairo Simon Da Fonseca E Martins, Gilberto De Andrade. Curso de estatística". $6^{\text {th }}$ Edição, editora Atlas - São Paulo (1996): 320.
7. Murray R. "Spiegel, Estatística Coleção Schaum". $3^{\text {rd }}$ Edição, Editora Afiliada, São Paulo (1993): 640.

8. Vieira Sônia. "Bio Estatística Tópicos Avançados". 2 $2^{\text {nd }}$ Edição, Editora Campus, Rio de Janeiro (2004): 212.

9. Vieira Sônia. "Introdução à Bioestatística". Rio de Janeiro: Campus (1991).

10. Maroco João. "Análise Estatística com utilização do SPSS". $2^{\text {nd }}$ Edição, Editora Silabo, Lisboa 508 págs (2003).

11. Alio Jl., et al. "Clinical outcomes after complete ring implantation in corneal ectasia using the femtosecond laser technology. A pilot study". Ophthalmology 118 (2011): 1282-1290.

12. Scarcelli Giuliano., et al. "Biomechanical Characterization of Keratoconus Corneas Ex Vivo with Brillouin Microscopy". The Association for Research in Vision and Ophthalmology 55.7 (2014).

13. Daxer A. "Biomechanics of corneal ring implants". Cornea JML 34.11 (2015).

14. Daxer A. "Corneal thickness after MyoRing implantation for keratoconus". The International Journal of Keratoconus and Ectatic Corneal Diseases 23 (2014): 15-19.

15. Spoerl E., et al. "Induction of cross-links in corneal tissue". EXperimental Eye Research 66 (1998): 97-103.

16. Hafezi Farhad., et al. "Pregnancy may trigger late onset of keratectasia after LASIK". Journal of Refractive Surgery 28.4 (2012).

17. De Araujo BS., et al. "Keratoconus progression after intrastromal corneal ring segment implantation according to age: 5-year follow-up cohort study". International Ophthalmology (2020).

18. Vega-Estrada A., et al. "Keratoconus Progression After Intrastromal Corneal Ring Segment Implantation in Young Patients: Five-year Follow-Up". Journal of Cataract and Refractive Surgery - LWW Journals 41.6 (2015): 1145-1152. 
19. Moscovici BK., et al. "Evaluation of keratoconus progression and visual improvement after intrastromal corneal ring segments implantation: A retrospective study". European Journal of Ophthalmology: SAGE Journals (2021): 11206721211000646.

\section{Assets from publication with us}

- Prompt Acknowledgement after receiving the article

- Thorough Double blinded peer review

- Rapid Publication

- Issue of Publication Certificate

- High visibility of your Published work

Website: www.actascientific.com/

Submit Article: www.actascientific.com/submission.php

Email us: editor@actascientific.com

Contact us: +919182824667 